\title{
Vertebral Artery Thrombosis after Spinal Injury: Case Report
}

\author{
Matthew J. Gambee, M.D. \\ Division of Physical Medicine and Rehabilitation, University of Utah Medical \\ Center, Salt Lake City, Utah, U.S.A.
}

\section{Summary}

The vertebral artery and the cervical spine are closely related anatomically, but damage to that vessel in cervical spinal injury and subsequent stroke involving the vertebrobasilar distribution has rarely been reported. A case of vertebrobasilar stroke following traumatic injury to the cervical spine is described. The anatomy of the vertebrobasilar system is reviewed and possible mechanisms of injury discussed. A literature review follows.

Key words: Spinal injury; Vertebrobasilar stroke; Vertebral artery thrombosis; Vertebral artery anatomy.

\section{Case report}

A 17-year-old male high school student was thrown from a bucking horse and landed on his head. He was rendered quadriplegic immediately. The patient was placed on a scoop, with sand bags used for cervical immobilisation and subsequently transported to a local hospital. Cervical spine $\mathrm{X}$-rays showed a comminuted fracture of C-5 with displacement of the body of C-5 posteriorly into the spinal canal. Clinical examination showed complete C-5 motor and sensory quadriplegia without other apparent injury. Following reduction of the fracture using Gardner-Wells tongs and seven pounds of cervical traction, the patient was air transported to our facility and admitted to the Neurosurgery Service.

Traction was subsequently increased to 15 pounds and the patient was cared for in a regular hospital bed being turned every 2 hours by the nursing staff. Six days post injury the patient suffered an apparent respiratory arrest requiring intubation and ventilatory support. Following resuscitation he remained comatose. A CT scan of the head, obtained the same day, showed dilatation of the lateral and third ventricles with displacement of the fourth ventricle anteriorly and to the right. There was a low density nonenhancing lesion in the left cerebellar hemisphere with upwards transtentorial herniation felt to be compatible with acute infarct and edema. Digital subtraction vertebral angiogram done shortly 
following the arrest revealed occlusion of the right vertebral artery at the C-5 level, an irregular and small basilar artery compatible with spasm and a paucity of vessels in the left cerebellar hemisphere. The left anterior and posterior inferior cerebellar arteries were not seen. An arch aortogram showed normal origin of the great vessels and dominant appearance of the left vertebral artery with a small right vertebral artery.

The patient remained comatose and was transferred to the Spinal Cord Injury Rehabilitation Service 8 weeks after the injury. Neurological examination of the cranial nerves revealed an inability to protrude the tongue, slight involuntary rotational movement of the neck, absence of corneal reflexes bilaterally, sluggish pupils, absence of gag reflex, slight facial grimace and disconjugate gaze with intermittent wandering horizontal nystagmus. The patient gave an inconsistent response to spoken commands by blinking his eyes. Diaphragmatic function appeared to be partially intact with spontaneous respiraions noted without ventilatory assistance for short periods. The patient remained ventilator dependent. No further progress was made toward neurological or functional recovery and therefore the patient was transferred to a hospital near his home four months later.

\section{Discussion}

\section{Anatomy}

The vertebral artery is normally the first and largest branch of the subclavian, arising deep in the neck from the cranial and dorsal surface of that vessel. It may be divided into four parts:

1. After arising from the subclavian arteries, it passes between the longus colli and the scalenus anterior to enter the transverse foramen of C- 6 .

2. The second part most commonly runs through the bony foramina of the first six cervical vertebrae. At the transverse process of the axis it runs laterally to the foramen in the transverse process of the atlas. Anatomical variation may occur with the artery passing in front of the transverse process of C-6 and entering the transverse foramen of C-5, C-4, or C-3.

3. After emerging from the transverse foramen of the atlas, the vertebral artery runs medially around the lateral articular process of the atlas. It then lies in a groove on the cranial surface of the posterior arch of the atlas and enters the vertebral canal by passing under the arch in the posterior atlanto-occipital membrane.

4. The fourth part pierces the dura mater and at the inferior border of the pons, it unites with the vessel of the opposite side to form the basilar artery. Branches of the vertebral artery include the anterior spinal artery and the posterior inferior cerebellar artery.

The basilar artery is a single trunk formed by the junction of the two vertebral arteries. The branches on either side of the basilar artery include the pontine branches, the labyrinthine artery, the anterior inferior cerebellar artery, the superior cerebellar artery and the posterior cerebral artery. 


\section{Pathogenesis}

There are a number of possible mechanisms of injury involved in damage to the vertebral artery following cervical spine trauma. Intimal tearing or damage with subsequent thrombus formation, intramural hematoma, dissecting aneurysm, or spasm with subsequent thrombus formation could all conceivably contribute to occlusion of the vertebral artery. Anatomical variation in circulation, underlying atherosclerosis, cervical spondylosis and cervical subluxations (congenital or traumatic) have been suggested as predisposing factors in vertebral artery thrombosis following chiropractic manipulation of the cervical spine (Krueger and Okazaki, 1980).

A number of interesting kinetic studies have demonstrated vertebral artery compromise with head movement. Toole and Tucker (1960) demonstrated interference of vertebral flow in 18 of 20 cadavers with rotational movements of the neck, most of them in the artery contralateral to the direction of movement. Hardesty et al. (1963) attached an electromagnetic flow meter to the vertebral artery exposed during radical neck dissection in two patients. There was reduction in flow of 23 percent in one patient and 9 percent in the other with head rotation to the contralateral side. Faris et al. (1963) showed evidence of vertebral artery occlusion with head positioning demonstrated by retrograde brachial arteriography in 43 asymptomatic volunteers. Occlusion occurred with contralateral rotation in three.

Three possible mechanisms of extraluminal vertebral artery obstruction with neck rotation have been described: (1) Compression of the artery by skeletal muscle or fascial bands at the junction of its first and second portions; (2) Compression throughout the second part by adjacent osteophytes; and (3) Compression or elongation of the artery by the sliding structures of the atlanto-axial joint (Krueger and Okazaki, 1980).

The mechanism of vertebral artery thrombosis in the case described above is not possible to determine precisely. The fact that thrombosis occurred unilaterally at the level of the fracture indicates that some type of injury to that artery occurred either acutely at the time of injury, or secondary to segmental instability of the damaged cervical spine at some period following hospitalisation. Routine patient positioning done with cervical traction in place may have caused enough rotation of the spine and subsequent extraluminal compression of an already damaged vertebral artery to result in complete thrombosis. Predisposing secondary factors of atherosclerosis, anatomical variation, and cervical spondylosis can be ruled out by the patient's age and the normal appearance of the anatomy on angiography following the event.

The question arises as to the etiology of an infarct in the left cerebellar hemisphere when only unilateral right sided vertebral artery thrombosis could be demonstrated by angiography. Possibilities include an embolic source originating from a pre-existing thrombus or perhaps a relative hypoperfusion state secondary to either hemodynamic compromise or basilar artery spasm resulting in ischemic infarction. Our patient sustained extensive damage in the region supplied by the posterior circulation in spite of a normal anterior circulation and apparent patency of the left vertebral artery as demonstrated by angiography. 


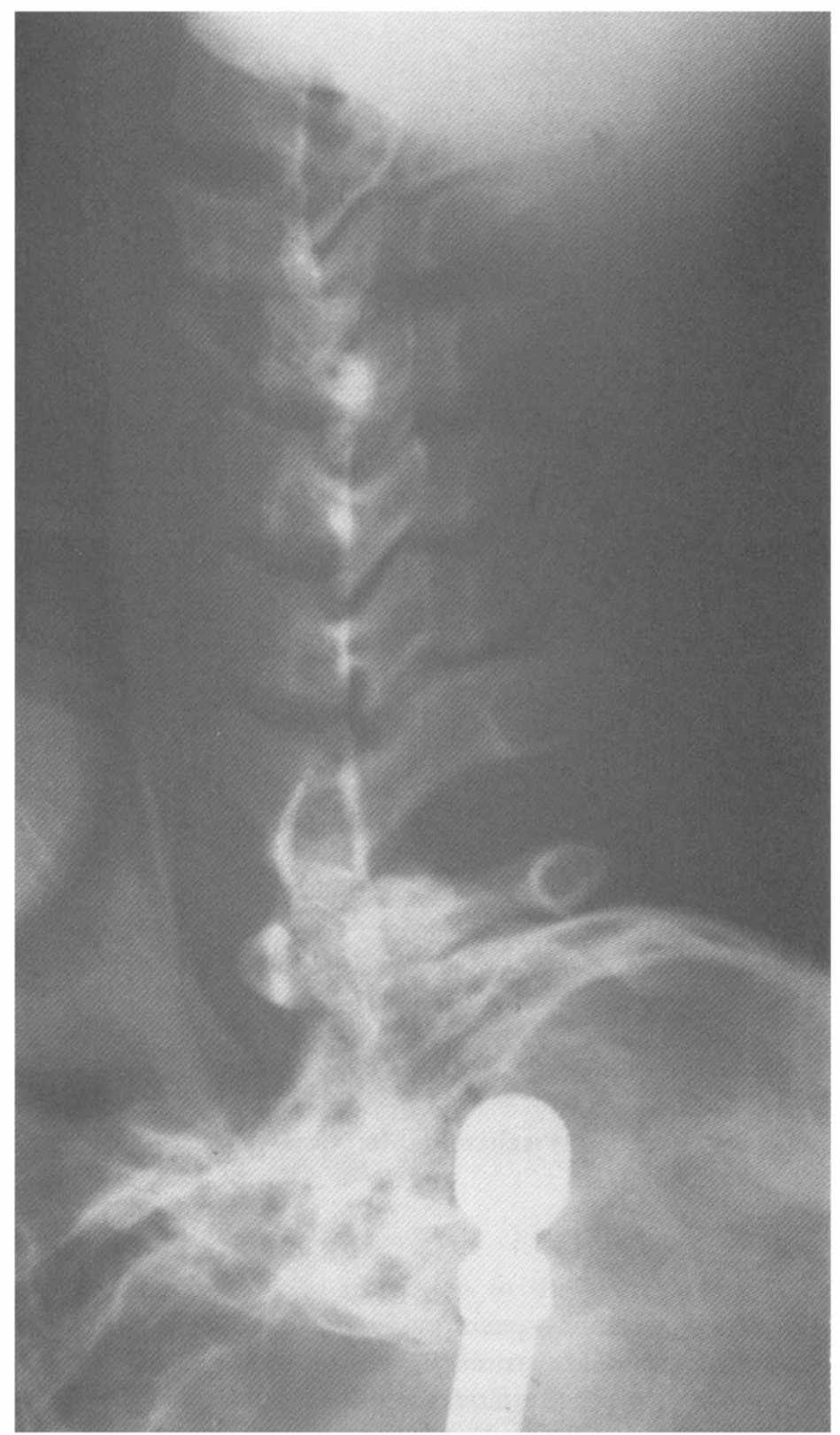




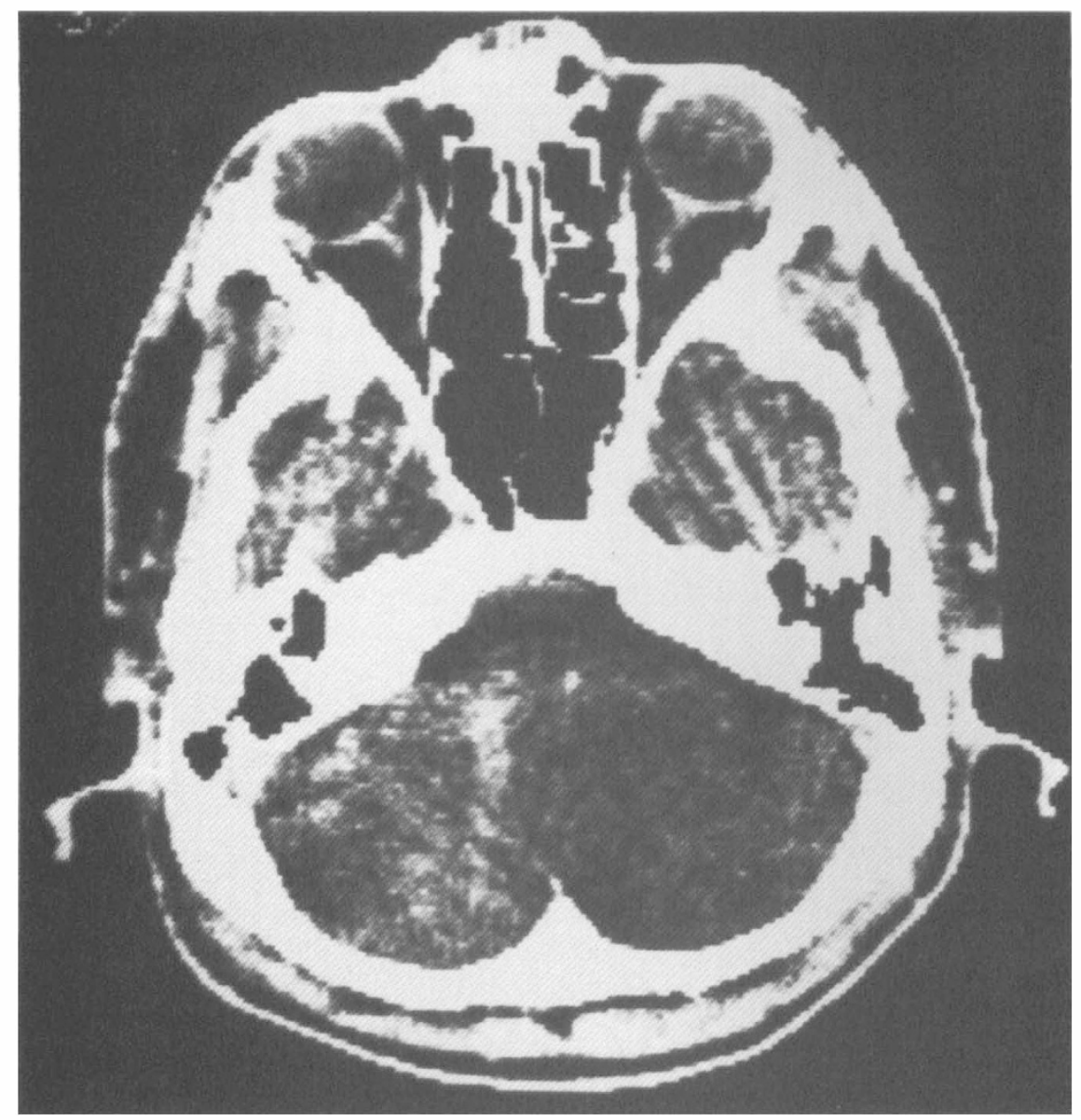

It is not clear whether subsequent ischemic infarction was secondary to embolus or thrombosis.

\section{Literature review}

Review of the literature revealed a paucity of reported cases of vertebral artery occlusion associated with spinal fractures. Suechting and French (1955) reported a case of posterior inferior cerebellar artery syndrome in a young man following a C-5/C-6 fracture-dislocation treated with cervical traction. Carpenter (1961) described a patient with vertebral artery thrombosis at the level of C-6 associated with fracture-dislocation of C-6/C-7. The patient died as a result of brainstem herniation following infarction of the cerebellum. Marks et al. (1973) described a case of C-5/C-6 fracture-dislocation following a football injury with immediate C-5 quadriplegia and cranial nerve deficits. Arteriogram in this case revealed occlusion of both vertebral arteries at the level of injury. Krueger and Okazaki 


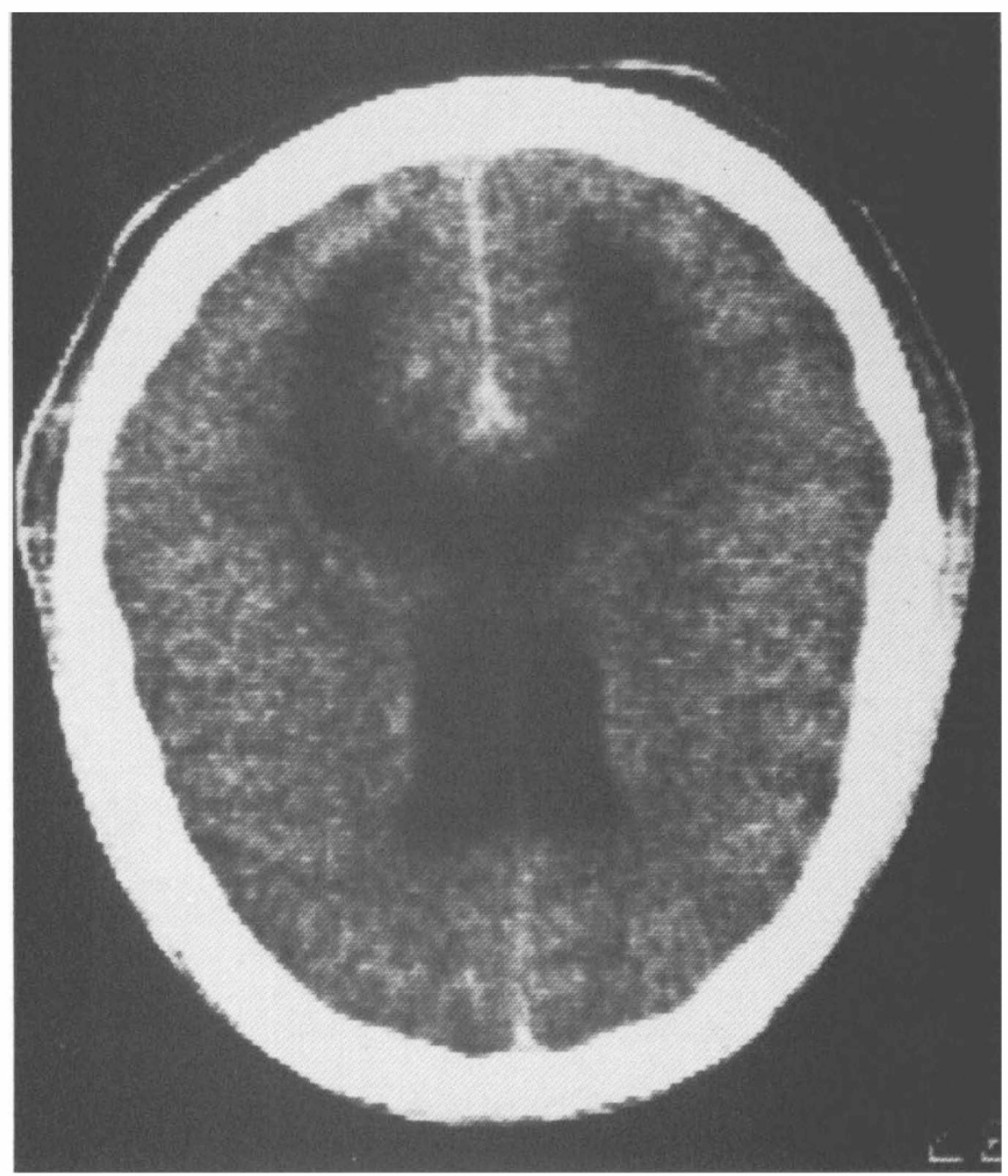

(1980) reported ten cases of infarction in the vertebrobasilar distribution in patients undergoing chiropractic manipulation, one of whom subsequently died. In total there have been approximately 50 reported cases of brainstem infarction following chiropractic cervical manipulation. Hayes and associates (1980) described a case of entrapment of the left vertebral artery at C-2/C-3 following blunt trauma to the anterior region of the neck. The entrapment resulted in aneurysm of the artery at the injury site which was treated with ligation of the artery at the C-1 level. Woolsey and Chung (1979) described a patient with C-5/C-6 fracture-dislocation who developed fatal basilar artery thrombosis 33 days after injury. At autopsy the left vertebral artery was thrombosed at the level of injury and the basilar artery was occluded throughout its entire length 
by the thrombus. Hughes (1964) reported two cases of cervical myelopathy following trauma to the cervical spine. There was no evidence of fracture in the cervical spine in either case but at necropsy there was kinking of the vertebral arteries in their intravertebral course which resulted in delayed onset of quadriparesis. Both patients died of related medical complications. Schneider et al. (1958) was the first to describe the central cord syndrome as a consequence of vascular injury involving the vertebral and anterior spinal arteries. Bose et al. (1985) described a case of transient ischemia in the distribution of the basilar artery following a traumatic cervical spine injury. Symptoms were completely reversed following acute heparinisation. The angiogram in this case showed bilateral vertebral artery occlusion at the level of the atlas. The collateral blood flow through the anterior circulation via the posterior communicating arteries undoubtedly prevented ischemic compromise of the vertebrobasilar distribution in this particular case.

\section{Conclusions}

A case of vertebral artery thrombosis following cervical fracture with subsequent ischemic injury in the vertebrobasilar distribution is presented. The anatomy of the vertebral artery was reviewed and possible mechanisms of injury discussed. It is not clear whether thrombosis or embolus was responsible for the ischemic cerebrovascular accident in this case. Previous reported cases have shown a similar pattern of traumatic injury to the cervical spine followed by late onset of ischemic damage in the vertebrobasilar distribution.

It is likely that injury to the vertebral artery is not a rare complication of spinal injury, but instead goes unreported or is not recognised because of sudden patient demise. Some patients may remain asymptomatic due to collateral circulation from the opposite vertebral artery or from the carotid circulation through the posterior communicating arteries. Our case appears to be unique in that the patient survived the insult.

Angiography is indicated in any patient with traumatic injury to the cervical spine who develops signs and symptoms of vertebrobasilar compromise. Acute anticoagulation resulted in marked clinical improvement in one reported case and should certainly be considered.

\section{References}

Bose B, NORTHRUP BE, OSTERHOLM JL 1985 Delayed vertebrobasilar insufficiency following cervical spine injury. Spine 10:108-110.

BRAUN IRA F et al. 1983 Brainstem infarction due to chiropractic manipulation of the cervical spine. Southern Medical Journal 76(9):1199-1201.

CARPENTER S 1966 Injury of the neck as cause of vertebral artery thrombosis. Journal of Neurosurgery $18-849$.

FARIS AA, POSER CM, WILMORE DW et al. 1963 Radiologic visualisation of neck vessels in healthy men. Neurology 13:386-396.

FRASER RAR, ZIMBLER SM 1975 Hindbrain stroke in children caused by extracranial vertebral artery trauma. Stroke 6:153-159.

GREEN D, JOYNT RJ 1959 Vascular accidents to the brainstem associated with neck manipulation. Journal of the American Medical Association 170:522-524.

GuARDJIAN ES, HARDY WG, KINDNER DW et al. 1963 Closed cervical cranial trauma associated with involvement of carotid and vertebral arteries. Journal of Neurosurgery 20:418-427. 
HARDESTY WH, WhitACRE WB, TOOLE JFet al. 1963 Studies on vertebral artery blood flow in man. Surgery, Gynecology Obstetrics 116:662-664.

HUGHES JT 1964 Vertebral artery insufficiency in acute cervical spine trauma. Paraplegia 9:2-13.

HuSNi EA, Bell HS, STORER J 1966 Mechanical occlusion of the vertebral artery: A new clinical concept. Journal of the American Medical Association 196:475-478.

HUSNI EA, STORER J 1967 The syndrome of mechanical occlusion of the vertebral artery: Further observations. Angiology 18:106-116.

KRUEGER BR, OKAZAKI H 1980 Vertebral-basilar distribution infarction following chiropractic cervical manipulation. Mayo Clinic Proceedings 55(5):322-332.

KUNKLE EG, MULLER JC, ODOM GL 1952 Traumatic brainstem thrombosis: Report of a case and analysis of the mechanism of injury. Annals of Internal Medicine 36:1329-1355.

LYNESS SS, WAGMAN AD 1974 Neurological deficit following cervical manipulations. Surgical Neurology 2:212-214.

MARKS RL et al. 1973 Nonpenetrating injuries of the neck and cerebrovascular accident. Archives of Neurology 28:412-414.

MEHALIC T, FARAT SM 1974 Vertebral artery injury from chiropractic manipulation of the neck. Surgical Neurology 2:125-129.

NyBERG-HANSEN R, LOKEN AC, TENSTAD O 1978 Brainstem lesion with coma for five years following manipulation of the cervical spine. Journal of Neurology, 218:97-105.

SCHNEIDER RC, Thompson JM, BeBIN J 1958 The syndrome of acute central cervical spinal cord injury. Journal of Neurology, Neurosurgery, Psychiatry 21:216.

SCHNEIDER RC, SCHemm GW 1961 Vertebral artery insufficiency in acute and chronic spinal trauma: With special reference to the syndrome of acute central cervical spinal cord injury. Journal of Neurosurgery 18:348-360.

SHEEHAN S, BAUER RB, MEYER JS 1960 Vertebral artery compression in cervical spondylosis: Arteriographic demonstration during life of vertebral artery insufficiency due to rotation and extension of the neck. Neurology 10:968-986.

SIMEONE FA, GOLDBERG HE 1968 Thrombosis of the vertebral artery from hyperextension injury to the neck: Case report. Journal of Neurosurgery 29:540-544.

SMITH RA, EstrIDGE MN 1962 Neurologic complications of head and neck manipulations: Report of two cases. Journal of American Medical Association 192:528-431.

SUECHTING RL, FRENCH LA 1955 Posterior inferior cerebellar artery syndrome following a fracture of the cervical vertebrae. Journal of Neurosurgery 12:187.

TATLOW WFT, BAMmER HG 1957 Syndrome of vertebral artery compression. Neurology 7:331-340.

TOOLE JF, TUCKer SH 1957 Influence of head position upon cerebral circulation: Studies on blood flow in cadavers. Archives of Neurology 2:616-623.

WoolsEy RM, CHUNG HD 1979 Fatal basilar artery occlusion following cervical spine injury.

Paraplegia 17(3):280-283. 